\title{
Correspondence
}

\section{How and to what extent can the humanities support oncology personnel? Could we devise a formula to guide policymakers?}

\section{Dear Sir,}

Scientific progress has profoundly changed the face of cancer: it is still a life-threatening disease but today, some types of cancer have a high chance of cure or at least of being lived with in the long term [1].

In the majority of cases, cancer patients survive while learning to live with certain level of uncertainty, and healthcare professionals follow the patient's journey sustaining their health-related and psycho-social related needs on the basis of their different clinical pathways.

Medical humanities are needed in medicine to deal with these aspects both from the patient and professional perspective.

At this regard, nowadays, national and international guidelines underline the importance of screening for patients' distress and subjective illness experience [2] but so far there has been a lack of guidelines addressing the psychological effects experienced by physicians, nurses and other members of the multidisciplinary team working with cancer patients [3].

Thus, it is mandatory that healthcare systems allocate resources to humanize oncology whereby healthcare professionals themselves may be helped to deal with cancer-related issues in a standardized way, not least because evidence from the literature underlines a high prevalence of burnout symptoms in healthcare staff in this area $[4,5]$.

\section{Reflection: which variables to be considered?}

As mental healthcare providers working every day in a comprehensive cancer center, we are drawn to wonder whether a formula could be propounded which policymakers could turn to in defining and allocating the proportion of medical humanities such as psychological care (i.e. empowerment interventions, relationshipand communication-focused workshops, Balint groups) that should characterize clinical pathways in different areas of oncology, from the point of view of healthcare providers.

Such a formula can also be related to institutional and budgetary activities dedicated to educating and empowering healthcare professionals.

We identified two related factors directly proportional to the level of humanization required.

The first factor we termed care load and it is specific to each oncologic area. It is related to the time spent caring for the patient by the team, to the number of outpatient examinations a patient has over a defined period of time, to the examination time, to the number of cancer treatments and diagnostic examinations and, in short, the overall relational time devoted to each patient by health care personnel.

The other factor is the intensity of the perceived illness threat.

This factor is mainly related to the perception that the patients have of the degree to which their life is threatened by their cancer and it is dependent on a number of factors: type of cancer, stage at diagnosis, previous familial negative experience with the same cancer, the social awareness regarding that specific type of cancer, the fact that one is in a surgical or medical context, the presence and the number of relapses, the presence of pain, and the impairment in quality of life.

For instance, in the surgical phase of early diagnosed breast cancer care, care-load is lower than in other specialties, surgical or medical ones: surgeons usually examine patients once and then operate on them and conduct follow-up. The hospital stay is usually no longer than three days and also the nursing of patients during hospitalization is not highly demanding from an emotional point of view. Also, in that specific surgical phase, the intensity of perceived illness' threat that patients have is low because in its early stages, breast cancer usually has a high chance of cure and this positively encouraging prognosis, related to early diagnosis, is usually communicated to the patients by healthcare professionals (surgeons) in order to promote their adjustment process before the histological characteristics of the tumor allow for personalized medical therapy. As a conclusion, the need for psychological attention that professionals have in this area is not so high as compared to other specialties.

For example, if we consider the medical oncology area which is employed in the cure of recurrent ovarian cancer, professionals' psychological needs and their care-load are very different.

Care-load is high because outpatients examinations are frequent, being conducted on a weekly or monthly basis, chemotherapy lines are multiple and relational issues are significant.

The intensity of perceived illness' threat in this area is also high and patients often share their fears with their physicians and nurses. When they ask questions, they are seeking not only answers but also support.

In these areas the need for humanization is higher and it is obvious to consider it crucial for professional wellbeing.

\section{Our proposal}

In our opinion, comprehensive cancer centers could map out their departments, units and wards at a programmatic level (as is the case with other workload indices) taking into additional consideration the personnel-related humanity index which is the combination of the care-load and the intensity of the perceived illness' threat in a specific oncology domain from the healthcare professionals point of view. 
This could allow for an amelioration of health care professional well-being including humanities not only in a patient-centered perspective but also from the point of you of care providers.

\section{References}

[1] K.D. Stein, K.L. Syrjala, M.A. Andrykowski, Physical and psychological long-term and late effects of cancer, Cancer 112 (11) (2008) 2577-2592.

[2] B.D. Bultz, L.E. Carlson, Emotional distress: the sixth vital sign-future directions in cancer care, Psychooncology 15 (2006) 93-95.

[3] A.E. Lowery, J.C. Holland, Screening cancer patients for distress: guidelines for routine implementation, Community Oncol. 8 (2011) 502-505.

[4] D.A. Whippen, G.P. Canellos, Burnout syndrome in the practice of oncology: results of a random survey of 1000 oncologists, J. Clin. Oncol. 9 (10) (1991) $1916-1920$.

[5] S. Banerjee, R. Califano, J. Corral, et al., Professional Burnout in European young oncologists: a European survey conducted by the European Society for Medical Oncology (ESMO), Ann. Oncol. 25 (5) (2014) 1-41.

Paola Arnaboldi*

Applied Research Division for Cognitive and Psychological Science, European Institute of Oncology, Via Ripamonti 435, 2014122063 Milano, Italy
Gabriella Pravettoni ${ }^{a, b}$

a Applied Research Division for Cognitive and Psychological Science, European Institute of Oncology,

Via Ripamonti 435, 2014122063 Milano, Italy

${ }^{\mathrm{b}}$ Department of Oncology and Hemato-oncology, University of Milan, Via Festa del Perdono 7, 20122

Milano, Italy

* Corresponding author. E-mail addresses: paola.arnaboldi@ieo.it (P. Arnaboldi), gabriella.pravettoni@ieo.it (G. Pravettoni).

26 February 2016

6 March 2016

Available online 11 March 2016 